A N N A L E S Annales de Bretagne et des Pays de l'Ouest

Anjou. Maine. Poitou-Charente. Touraine

$112-4$ | 2005

Varia

Politique, religion et affaires : Pierre Assézat (vers 1515-1581)

\title{
Francis Brumont
}

\section{(2) OpenEdition}

Journals

Édition électronique

URL : http://journals.openedition.org/abpo/1051

DOI : 10.4000/abpo.1051

ISBN : 978-2-7535-1500-0

ISSN : 2108-6443

\section{Éditeur}

Presses universitaires de Rennes

Édition imprimée

Date de publication : 20 décembre 2005

Pagination : 147-156

ISBN : 978-2-7535-0243-7

ISSN : 0399-0826

\section{Référence électronique}

Francis Brumont, «Politique, religion et affaires : Pierre Assézat (vers 1515-1581) », Annales de

Bretagne et des Pays de l'Ouest [En ligne], 112-4 | 2005, mis en ligne le 20 décembre 2007, consulté le 19 avril 2019. URL : http://journals.openedition.org/abpo/1051 ; DOI : 10.4000/abpo.1051 


\title{
Politique, religion et affaires : Pierre Assézat (vers 1515-1581)
}

\author{
Francis BRUMONT \\ Professeur d'histoire moderne \\ Université de Toulouse 2
}

Lorsque, en 1555, Pierre Assézat commence à faire édifier le magnifique hôtel qui a perpétué sa mémoire jusqu'à nous, il peut estimer que la fortune l'a comblé de ses faveurs; il ne lui a même pas manqué ce petit coup de pouce du destin qui lui a permis de se retrouver chef de famille assez jeune, à cause de la mort prématurée de ses frères aînés ${ }^{1}$. En peu de temps, sa position sociale change; il se retrouve parmi les notables de la cité, position encore confortée par l'importance de ses affaires qui le conduisent à fréquenter de grands marchands internationaux de premier plan. La construction de son hôtel s'inscrit dans une suite logique et est en quelque sorte le symbole de son ascension sociale et la marque visible de sa nouvelle position. Mais cette situation fut totalement compromise et la vie de Pierre Assézat prit un cours nouveau à la fin des années 1550 lorsqu'il se convertit au calvinisme, une décision sans doute mûrement réfléchie, même si elle a pu être favorisée par l'ambiance de l'époque, qui est celle où les conversions se multiplient, notamment parmi les élites urbaines ${ }^{2}$.

Il est certain que cet événement d'ordre privé, intime même, n'a pas eu de conséquence immédiate dans la vie de Pierre Assézat, notamment en ce qui concerne ses affaires. Mais, rapidement, la tournure prise par les événements au niveau national, les passions exacerbées, les premiers incidents, puis les débuts de la guerre civile, ne purent qu'interférer dans la vie d'une personnalité éminente comme celle d'Assézat. Et effectivement, il ne tarda pas à s'impliquer personnellement, suite à son élection, début

1. Sur tout ce qui suit, je renvoie une fois pour toutes, à BRUMONT, Francis, "Pierre Assézat, un marchand dans son siècle ", dans Peyrusse, Louis, Tollon, Bruno (dir.), L'hôtel d'Assézat, Toulouse, Association des Amis de l'hôtel d'Assézat, 2002, p. 37-76 et à GiNESTY, Henry, "La famille d'Assézat d'après les archives notariales (XVI p. 155-178, où l'on trouvera toutes les références.

2. Pour un résumé commode sur la Réforme en France et les guerres de religion, JoUANnA, Arlette et al., Histoire et dictionnaire des guerres de religion, Paris, Robert Laffont, coll. "Bouquins ", 1998, 1526 p. 
1562, au capitoulat, en compagnie de sept autres de ses coreligionnaires. C'est alors que sa vie bascula, qu'il connut l'exil et la prison, sans cesser de s'impliquer dans la défense de ses convictions et de son " parti ". C'est l'intrusion de la politique dans la vie de cet homme que nous allons étudier ici en montrant tout d'abord quelle place enviable il avait atteint dans ses affaires et la société toulousaine et même au-delà, puis nous analyserons rapidement les événements de mai 1562 qui le conduisirent à l'exil et enfin, nous verrons comment, malgré les vicissitudes de sa vie personnelle, Pierre Assézat ne cessa jamais, et de faire des affaires, et de soutenir la cause protestante, peut-être même après sa conversion de septembre 1572.

En 1545 et 1546, décèdent successivement Bernard et Noël Assézat, marchands de Toulouse. C'est surtout, semble-t-il, Noël qui brasse les affaires les plus importantes; il est d'ailleurs associé avec son cadet Pierre, dans une société dont le capital atteint 75000 livres, dont les deux tiers lui appartiennent. Par son testament, Noël, qui a trois enfants légitimes en bas âge, institue son beau-frère Pierre Delpech, marchand lui aussi, et Pierre Assézat comme tuteurs, mais réserve le maniement des capitaux appartenant aux orphelins à son seul frère, au grand dépit de Delpech qui entame assez rapidement, dès 1550, une série de procès contre son cotuteur. Voilà donc Pierre, par les hasards de la démographie, à la tête des affaires familiales et nanti d'un capital important, qu'il n'aurait rêvé d'accumuler en si peu de temps, s'il avait dû rester au service de ses frères.

Cette situation économique enviable devait s'affirmer sur le plan social : c'est chose faite en 1548 lorsqu'il épouse Peyronne de Cheverry, issue de deux familles des plus notables de la cité, les Cheverry et les Lancefoc. Il ne tarde pas à toucher les dividendes de cette inclusion dans le cercle étroit des " grandes " familles en accédant au capitoulat en 1551, ce qui lui confère ipso facto la noblesse. Dès sa fondation en 1549 il fait partie de la bourse des marchands de Toulouse dont il fut prieur à plusieurs reprises et c'est sans doute à ce titre qu'il fut appelé par Henri II à Paris pour conférer avec lui sur les moyens de remédier à la crise qui frappait le commerce du royaume à cette époque.

Assézat est avant tout un marchand de pastel qu'il expédie en grandes quantités en Espagne, aux Pays-Bas, en Angleterre, à Rouen... dans les années 1550, on peut estimer à 180000 livres le chiffre d'affaires généré annuellement par cette seule activité. Elle le met en relation avec les plus grands marchands internationaux, en particulier à Anvers, où il est en affaires avec Gaspard Schetz, Pierre Moucheron, Christophe Pruynen, Gillis Hooftman, c'est-à-dire avec l'élite des commerçants de la grande cité brabançonne. Mais Assézat ne se contente pas du commerce; comme ses pairs, il se livre aussi à des opérations financières dont les plus simples sont les prêts d'argent, les assurances, l'affermage de divers revenus. Son plus beau " coup " dans ce domaine est la prise à ferme d'une partie des revenus de la reine douairière Éléonore, veuve de François ${ }^{\mathrm{er}}{ }^{\text {, }}$ puis de ceux de sa fille, Marie de Portugal, qui en hérite en 1558. Cette affaire est intéressante, car 
elle porte sur des sommes importantes et montre l'étendue et la variété des relations d'Assézat; c'est, en effet, grâce à Gaspard Schetz qu'il obtient d'affermer les revenus de la reine dans le sud-ouest de la France. Par ailleurs, la maison française de la princesse Marie est dirigée, au moins à partir de 1560, par son chancelier, Michel Dufaur, président au parlement, qui a été suspecté de calvinisme et, de fait, tous ceux qu'il nomme pour s'occuper des affaires de la princesse appartiennent à la nouvelle religion : son secrétaire, Jean du Portal (qui sera décapité après les événements de 1562), son procureur, Barthélemy Prévost, son trésorier, le maître des eaux et forêts Jean de Saint-Étienne, et, enfin, les fermiers des droits, Assézat et son neveu Antoine Cros. Ces nominations ne peuvent résulter que d'une décision délibérée et ne doivent rien au hasard. En s'insérant dans la clientèle des Dufaur, Assézat s'assurait un soutien politique puissant, qui confortait la position éminente que sa richesse lui assurait. Il est certain aussi que dans les années 1550 la répression contre les réformés sévit à Toulouse et que ceux de la nouvelle religion ont tout intérêt à se serrer les coudes ${ }^{3}$.

Toujours est-il que celle-ci n'atteint pas Pierre Assézat avant le début des guerres de religion, mais nous ne savons pas à partir de quand il a manifesté son appartenance au calvinisme, les nominations dont nous avons parlé plus haut n'intervenant qu'en mai 1560, date à laquelle il est probable que les nouvelles idées d'Assézat sont connues. En juillet de cette même année 1560, il fait partie (avec notamment A. Cros et J. de Saint-Étienne) de la troupe des protestants qui tente d'envahir le Capitole ${ }^{4}$. En 1562, les élections au capitoulat portent au pouvoir huit édiles protestants, parmi lesquels Pierre Assézat; c'est ce que Georges Bosquet nomme la " création impie et désastrée des capitouls nouveaux ${ }^{5}$ ".

À partir de ce moment-là, la tension est palpable dans la ville; le 4 avril, des incidents éclatent au faubourg Saint-Michel lors de l'enterrement de la femme catholique d'un charpentier huguenot, suivis du pillage de certaines maisons appartenant à des protestants. Le même jour, des membres du parlement se rendant au Capitole y trouvent des huguenots retranchés, "armés comme dans une forteresse ${ }^{6}$ ". Quel est le rôle d'Assézat lors de cette montée des périls? Il est certainement de premier plan : c'est du moins ce qui apparaît à travers la lecture de l'opuscule de Georges Bosquet. Le 4 avril, avec son collègue capitoul, Ganelon, il fait déposer les armes à ses coreligionnaires; le lendemain, il fait partie de la réunion de conciliation qui se

3. Sur cette répression, JouAnNA, Arlette et al., La France de la Renaissance. Histoire et dictionnaire, Paris, Robert Laffont, coll. " Bouquins », 2001, p. 336-341.

4. Bosquet, Georges, Histoire de M. G. Bosquet sur les troubles advenus en la ville de Tolose l'an 1562, Toulouse, R. Colomiez, 1595, p. 29. Cette édition est la traduction en français de l'ouvrage de Bosquet paru en latin en 1563; l'auteur est un catholique exalté, si bien que son ouvrage fut condamné à être brûlé par arrêt du conseil privé le 18 juin 1563 , mais il a été témoin des faits qu'il rapporte et à ce titre peut être utilisé avec les précautions d'usage.

5. Ibidem, p. 40.

6. Ibid., p. 67-68. 
tient au monastère des Augustins les catholiques étant dirigés par le premier président du parlement, Jean de Mansencal. Le 9 mai, avec son collègue Mandinelli, il conduit les négociations de paix entre les deux parties, à un moment où les tensions s'exacerbent; le 11 mai, avec son neveu Antoine Cros et deux autres huguenots, il présente les articles de cette paix, qualifiée d'" androgine " par Bosquet, devant une vaste assemblée populaire $^{7}$. Mais il est déjà trop tard : la méfiance est générale et réciproque; dès le 9 mai Monluc avait averti Mansencal que les huguenots avaient reçu ordre de Condé de s'emparer de la ville le 18 de ce mois ${ }^{8}$. Il est probable que c'est la réaction attendue des catholiques qui poussa les amis d'Assézat à avancer la date de leur opération : c'est le 11 mai, vers 9 heures du soir, qu'ils s'emparèrent de l'hôtel de ville et de quelques endroits stratégiques. Au bout de quelques jours de combat, la défaite des insurgés fut consommée et le 17 mai ils durent abandonner la ville. Assézat put s'enfuir comme les autres capitouls, à l'exception d'un seul qui fut exécuté, et se réfugia peutêtre à Montauban que Monluc partit assiéger, mais ne put prendre.

Rappelons que les événements de mai 1562 s'inscrivent dans le cadre plus large de la révolte des troupes huguenotes dirigées par le prince de Condé en réaction au massacre de Wassy ${ }^{9}$. La prise de Toulouse eût été pour les réformés d'une importance capitale, car elle aurait fortement consolidé leur domination sur la vallée de la Garonne et le Languedoc. Leur défaite est due à la vive réaction de leurs adversaires, dûment avertis, à l'impossibilité de ce fait de recevoir les secours qui devaient arriver de Montauban et à l'opposition ferme des parlementaires; notons que les principales villes, Bordeaux, Aix et Toulouse, où les tentatives des huguenots échouèrent étaient sièges de parlement.

À partir de ce moment, la vie d'Assézat bascule, même si l'arrêt du 18 juin 1563 le rétablit, lui et les autres capitouls, dans toutes ses prérogatives; comptant trop d'ennemis dans la ville, étant en butte aux tracasseries des juges (refus du parlement d'enregistrer l'édit, confiscation de ses livres et autres papiers) et, en général, de ses ennemis, il ne se sentait pas en sécurité à Toulouse où il ne vint que sporadiquement, durant les périodes de trêve. C'est que ces ennemis, et surtout les plus acharnés, étaient des membres de sa propre famille. Celle-ci, comme bien d'autres, était, en effet, divisée sur le plan religieux: Peyronne de Cheverry était restée catholique comme leurs enfants et s'occupa du mieux qu'elle put des affaires de son mari alors que son propre frère, Pierre de Cheverry, trésorier général des finances, avait lui aussi rejoint les rangs de la Réforme. Ce n'était donc pas de ce côté que pouvait venir le danger, mais bien plutôt d'un vieil adversaire d'Assézat, son beau-frère et cotuteur, Pierre Delpech, dont nous avons

7. Tout ceci d'après BosQuET, Georges, op. cit., p. 67-68, 86-87, 91.

8. Ibidem, p. 88; sur la chronologie précise des actions de Monluc, Courteault, Paul, Blaise de Monluc historien. Étude critique sur le texte et la valeur historique des Commentaires, Toulouse/Paris, Privat/Picard, 1908, p. 425-431.

9. Jouanna, Arlette et al., Guerres de religion..., op. cit., p. 114-120. 
déjà noté qu'il avait sans doute mal supporté que le testament de Noël ait réservé l'utilisation des capitaux des orphelins à Pierre Assézat.

Pierre Delpech est un catholique intransigeant et l'éloge qu'il mérite de la part de Georges Bosquet, qui l'est tout autant, ne laisse planer aucun doute à ce sujet : "Pierre Delpech, citoyen très fidelle et zélé catholique [...] entendu à trafique, versé ès bonnes lettres, médiocrement riche, prudent, vaillant et hardi, grand ennemy des hérétiques ${ }^{10}$. " Et Pierre Delpech ne se contente pas d'être un zélé catholique, il agit : le même Bosquet nous apprend qu'il entretient chez lui " puys trois mois à ses despens bon nombre de soldats ${ }^{11}$ ", une milice qu'il utilise à bon escient puisque dès le 11 mai, le « capitaine » Delpech est à la tête de ceux qui résistent aux huguenots soulevés. Il est nommé capitoul à la place des protestants destitués en 1562 en compagnie d'autres catholiques aussi zélés que lui et délégué aux États du Languedoc l'année suivante ${ }^{12}$. En 1566, son fils est nommé par les capitouls capitaine de 200 argoulets destinés à surveiller les protestants ${ }^{13}$ et durant les années suivantes Delpech est toujours présent quand il s'agit d'acheter de la poudre, de payer des soldats, de dénoncer les manigances des hérétiques, de préparer des coups de main contre les cités tenues par les protestants ${ }^{14}$. Mais surtout, son rôle est capital lors des massacres de la "Saint-Barthélemy " toulousaine, les 3 et 4 octobre 1572. Revenant de Paris avec son collègue P. Madron, il prétend avoir un ordre oral du roi pour faire massacrer les protestants que les autorités avaient fait emprisonner pour les protéger. Malgré l'opposition du parlement, il réunit chez lui une troupe de 60 à 80 hommes, pour la plupart des notables, (dont un fils ou neveu d'Assézat), qui se livrent au massacre des huguenots retenus au couvent des Jacobins ou dans les prisons et au pillage de leurs maisons ${ }^{15}$.

On voit donc que Toulouse n'était pas un lieu très sûr pour Pierre Assézat; les autorités multiplient les mesures à l'encontre des huguenots, confiscant leurs biens, les assignant à résidence. Les métairies d'Assézat sont pillées en septembre 1568 et, en cette même année, il fait partie des protestants qui durent payer une taxe de 30000 livres : sa part s'éleva à 2500 livres, un peu moins que celle de son ancien collègue capitoul de 1562 Antoine Ganelon (3000 livres), mais plus que Michel Dufaur (2000 livres), ou que son beau-frère Cheverry et son neveu Cros (1000 livres chacun). Ce serait mal connaître Assézat cependant que de croire qu'il subissait ces événements sans réagir; il avait d'autres armes que la simple violence pour lutter en faveur de ses coreligionnaires : ces armes, ce sont les finances, le nerf de la guerre.

10. BosQUET, Georges, op. cit., p. 91 .

11. Ibidem.

12. Devic, dom C. et VAISSETE, dom J., Preuves de l'Histoire du Languedoc, éd. de 1889, Toulouse, Privat, tome XII, col. 649 et 654 .

13. Ibidem, col. 862 .

14. Ibid., col. 872, 921, 950, 995 (1568-1572).

15. Ibid., col. 1023-1026. 
Ce sont les registres des écrous de la Conciergerie de Paris qui nous apportent quelques éclaircissements sur "l'existence d'une internationale de marchands favorables au parti protestant ${ }^{16}$ ". Parmi les écroués figure, en effet, François Milhau, facteur d'Assézat, qui, en 1560, l'avait envoyé à Rouen (et dans le Nord en général) pour s'y occuper de ses affaires. Il avait dû s'installer à Paris où il avait une maison qui fut fouillée (et pillée) lors de son arrestation : on y trouva des promesses de prêts sans intérêt en faveur du prince de Condé, pour un montant de deux millions de livres. Il est probable que cette arrestation faisait suite au procès intenté à Bruxelles par le duc d'Albe contre Assézat; en effet, en même temps que Milhau, écroué le 5 janvier 1569, furent arrêtés François Marchant (4 janvier) et Guillemette Chauvet (8 janvier), dont l'écrou dit explicitement que c'est dans le cadre des poursuites contre Assézat.

Ce sont ses relations avec les grands marchands d'Anvers qui ont valu à Assézat l'honneur de figurer parmi les milliers d'opposants à Philippe II poursuivis par le duc d'Albe par l'intermédiaire du Conseil des troubles. Gaspard Schetz faisait partie de ces opposants, mais c'était plutôt un modéré, un de ceux que l'on aurait appelés en France un " politique "; il s'opposa surtout au duc d'Albe lorsque celui-ci voulut imposer le "dixième " une taxe sur la circulation des marchandises (décembre 1569). Ce n'était pas le cas de Gillis Hooftman et de Pierre de Moucheron, calvinistes convaincus, qui soutinrent la révolte de leurs forces et de leurs deniers. Les deux pouvaient se prévaloir de l'amitié du prince d'Orange et de bien d'autres personnages comme Thomas Gresham ou Christophe Plantin. Moucheron était aussi lié avec Pierre de la Place, premier président de la Cour des Aides de Paris et agent du prince de Condé ${ }^{17}$. Du prince de Condé au prince d'Orange, l'internationale des marchands finançait ainsi, à des conditions avantageuses sans doute, l'internationale protestante. Pierre Assézat devait y jouer un rôle digne de son envergure, aux côtés de marchands lyonnais ou parisiens dont certains eurent moins de chance que lui, puisque Nicolas Croquet et Philippe de Gastine, écroués eux aussi le 5 janvier 1569, furent exécutés " pour leur soutien financier au parti de Condé ${ }^{18} "$.

Assézat dut en éprouver une frayeur rétrospective, lui qui avait été décrété d'arrestation un peu plus tard, le 19 février, et qui fut écroué au Château-Trompette, à Bordeaux. François Milhau, quant à lui, avait été arrêté la veille " en prévention d'avoir ouy ministres à Paris, pourté les

16. BOUCHER, Jacqueline, « Les incarcérations à la Conciergerie de Paris pour fait de religion, 1567-1570 ", dans ChEVALIER, Bernard, SAUZET, Robert (dir.), Les Réformes : enracinement socio-culturel, Paris, 1985, p. 313.

17. Pierre de la Place a laissé plusieurs ouvrages dont les Commentaires de l'estat de la religion et république soubs les rois Henry et François seconds et Charles neufviesme qui couvrent la période 1556-1561; il fut massacré lors de la Saint-Barthélemy (BucHON, J. A. C., Choix de chroniques et mémoires sur l'histoire de France avec notices biographiques, Paris, Desrez, 1836, p. IX-XII).

18. BOUCHER, J., art. cit., p. 314. 
armes à Orléans et à Lion aux premiers troubles de l'an mil cinq cens soixante deux ${ }^{19}$ ". Cet épisode de la vie d'Assézat est rappelé dans les lettres patentes que le roi lui octroya en 1572, à la suite de son abjuration : " sans qu'il y eust aulcunes charges et information contre luy [il a] esté puys naguerre emprisonné au château Trompette de notre ville de Bordeaulx et tous ses biens et marchandises saisis et arrestés en notre dite ville de Toulouse. Or est-il que notre aimé et féal chevalier de notre ordre le sieur de Monpezat notre lieutenant général au gouvernement de Guyenne s'estant despuis informé de la cause des dits emprisonnements et saisie et ayant cogneue la calompnye des hayneux du dit suppliant et son innocence l'auroit eslargy par la ville ${ }^{20} »$. Melchior de Montpezat, gendre du marquis de Villars, remplaça souvent ce dernier nommé lieutenant général de Guyenne (à la place de Monluc) le 3 septembre $1570^{21}$. Est-ce à dire que c'est suite à la paix de Saint-Germain (8 août 1570) qu'Assézat fut élargi ? Auquel cas il serait resté un an et demi en prison, ce qui expliquerait son absence dans la documentation à cette époque.

Toujours est-il que les « haineux " qui poursuivent Assézat de leur vindicte et dont certains ne nous sont pas inconnus auront raison de sa volonté : il fut contraint à l'abjuration, comme tant d'autres, cérémonie qui se déroula en l'église Saint-André de Bordeaux, le 30 septembre 1572, à l'issue de la grand-messe. Le roi par ses lettres patentes du 15 octobre suivant le rétablit dans tous ses biens et dignités, mais il ne revint que rarement à Toulouse, résidant plutôt à Bordeaux d'où il pouvait contrôler plus facilement ses affaires et, notamment, la vente du pastel.

Même aux moments les plus sombres, Pierre Assézat ne cessa jamais d'être un marchand et un financier. À partir de 1561, le commerce du pastel avec les pays du Nord, et en particulier les Pays-Bas, était en fort déclin à cause des difficultés politiques et industrielles d'Anvers et de la concurrence de l'indigo. La crise, cependant, ne se manifesta que sporadiquement au début, car les arrivées étaient tributaires des récoltes d'une plante dont on maîtrisait mal la culture et du transport par les flottes espagnoles. Si bien que certaines années ce produit fait défaut, ce qui redonne toutes ses chances au pastel comme en 1574-1575 où Assézat pense envoyer de grandes quantités en Espagne et faire de gros bénéfices : «Espaire fère plus de besoinhes en ung an que james je haye fette en ma vie " écrit-il à son correspondant de Pampelune, le 4 septembre $1575^{22}$. Ses espérances seront déçues, surtout à cause de la guerre qui rend difficile et onéreuse la navigation sur la Garonne et parce que cette pénurie était conjoncturelle. Toujours est-il que c'est vers l'Espagne, la Navarre surtout, qu'Assézat a replié ses affaires commerciales; le pastel continua, en effet, à être utilisé

19. Ibidem, p. 79 .

20. Arch. mun. de Toulouse, AA 15, $\mathrm{n}^{\circ} 211$.

21. Blaise de Monluc, Commentaires, Paris, Gallimard, 1964, p. 1297 et 1388.

22. BRumont, Francis, LARguIER, Gilbert, "Trois lettres marchandes de Pierre Assézat (1575) ", Annales du Midi, tome 110, n²22, 1998, p. 242. 
dans ce pays, conjointement avec l'indigo, pendant un certain temps encore, mais le montant de ces affaires est bien inférieur à ce qu'il était avant 1561 : on peut calculer qu'alors le chiffre d'affaires du seul pastel atteignait, bon an mal an, les 200000 livres tournois alors que ce ne sont que quelques dizaines de milliers de livres qui sont concernées par les échanges avec Pampelune.

Cependant, cela ne signifie pas que comme bon nombre d'autres marchands, Assézat se soit replié sur ses terres et ait mené une vie de rentier. C'est vers les affaires financières qu'il se tourne; nous avons déjà parlé de l'afferme des revenus de la reine Éléonore et de sa fille la princesse Marie de Portugal; le 20 février 1567, il lui devait 4700 et quelques livres, qu'il n'avait pas encore payées dix ans plus tard, si bien que le parlement le condamna à la rembourser avec les intérêts qui s'élevaient à plus de 3000 livres. Cela signifiait qu'il continuait à gérer ces revenus, mais ce n'était pas les seuls. En février 1564, il prit en sous-afferme d'un banquier de Lyon les droits levés sur les procès; il s'engage pour 48000 livres : nous retrouvons donc là des chiffres dignes de lui, même s'il semble qu'ensuite il ait cédé ses droits à son cousin Pierre Lancefoc, à François Chalvet et à d'autres. Peu de temps après, il se charge de collecter les revenus de l'évêché de Comminges, pour Charles de Bourbon, baron de Carmaing, fils illégitime du feu roi de Navarre, sans doute parce que celui-ci lui devait 3000 écus.

Mais, ce n'était là que petites affaires; il est bien probable que la prise à ferme des revenus du roi de Navarre en Guyenne, contrat dont il jouit en compagnie de Charles d'Aste, conseiller du roi et comptable de Bordeaux, a été de bien plus d'importance. C'est le 24 juin 1573 que les deux associés avaient pris cette ferme pour une durée de neuf ans. Nous n'en connaissons pas le montant, mais en 1575, le roi de Navarre les somme de racheter les terres de Chalus et de Sainte-Bazeille qui avaient été aliénées pour 94000 livres. En 1576, ils sous-afferment une partie de ce domaine, les recettes d'Albret, Armagnac et Fezensaguet pour 7 ans et 72222 livres par an. Nous n'en savons pas plus sur cette importante affaire; mais, la signature de ce contrat signifie que même après son abjuration Pierre Assézat faisait toujours parti des fidèles du roi de Navarre. Sans doute avait-il participé aussi plus directement au financement de la cause, comme semblent le montrer les accusations qui sont portées contre lui à Bruxelles et suite au pillage de la maison parisienne de François Milhau. Il ne faut pas, en effet, croire ce que déclarent les lettres patentes qu'il obtient après son abjuration où le roi précise qu'il n'a pas contrevenu aux ordonnances, ni " onques donné conseil, ny ayde de force ou d'argent, ne de choses que ce soit à ceulx qui ont tenu party contraire à lui ". C'est là pure rhétorique pour justifier la réconciliation d'Assézat.

En conclusion, je signalerai simplement que la religion n'interfère pas dans les affaires : les relations commerciales n'entrent pas dans la sphère du privé et Assézat continue à traiter avec son correspondant à Pampelune Sancho d'Abaurrea après que les événements de 1562 ne permettent pas 
le doute sur son appartenance religieuse. Et à un niveau plus général, Pampelune a des relations économiques privilégiées avec La Rochelle comme Oloron en Béarn traite habituellement avec Saragosse sans que cela entraîne des difficultés autres que conjoncturelles, lorsque l'Inquisition décide de surveiller plus attentivement les marchands étrangers. De même, les correspondants anversois d'Assézat sont indifféremment catholiques ou protestants, comme ils sont d'origine italienne, allemande ou française. Cela ne semble poser de problèmes à personne et il n'y pas lieu de s'en étonner : Pierre Jeannin fait remarquer que Philippe II " traite avec les Hanséates tels qu'ils sont, hérétiques ${ }^{23}$ ".

Cependant, la rupture religieuse a sans doute resserré les solidarités et il est des affaires que l'on réserve à des amis sûrs et pas uniquement celles qui concernent les finances des coreligionnaires ou la fourniture d'armes ou de munitions. Il est logique que l'afferme des revenus du roi de Navarre soit confiée à des réformés (ou à de récents convertis) et que l'on fasse profiter les amis des meilleures affaires, témoin la composition de la maison de la princesse du Portugal à Toulouse. De nombreux liens de ce type doivent pourtant nous échapper, car le maximum que nous pouvons connaître (et encore) c'est la confession des intéressés, et non les nombreux autres liens, qui vont des sentiments personnels d'amitié ou d'inimitié jusqu'aux liens de parenté et de clientèle que l'on peut parfois soupçonner. Et dans ces cas, il est difficile de savoir quelle relation prend le pas sur les autres, les guerres civiles, et les guerres de religion en particulier, fournissent de nombreux exemples où les liens du sang, de clientèle, de fidélité prennent le pas sur l'appartenance religieuse ou la conditionnent. Pour en revenir à notre personnage, il est fort probable que sa conversion - forcée - n'a pas entamé les liens de solidarité qui l'unissaient au parti protestant et qu'il a continué de participer à son financement. Quelques éléments dans son testament, comme l'absence d'invocation des saints et de la Vierge ${ }^{24}$, montrent qu'il n'a peut-être pas tout renié de son ancienne religion, ce qui s'accorderait assez bien avec sa conduite prudente durant les dernières années de sa vie.

23. JEANNIN, Pierre, Les marchands au XVI siècle, Paris, Seuil, 1957, p. 160.

24. GINESTY, Henri, « La famille d'Assézat... ", art. cit., p. 176. 


\section{RESUME}

Grâce à un heureux concours de circonstances, P. Assézat se retrouve vers 1550 à la tête d'une fortune et d'une situation sociale enviables. Mais sa conversion au calvinisme et sa participation active au soulèvement huguenot de 1562 le contraignent à l'exil et l'exposent à des représailles (emprisonnement). Cependant, cela ne l'empêche pas de continuer à soutenir la cause réformée de ses deniers et grâce au réseau de relations internationales que son activité marchande lui avait permis de constituer, notamment aux Pays-Bas. Même après son abjuration en 1572, il continue à faire partie des fidèles du roi de Navarre. Et pendant tout ce temps, il ne néglige pas ses affaires, la vente du pastel notamment, affaires qu'il traite indifféremment avec des protestants ou des catholiques, se conformant ainsi à la pratique habituelle de ses pairs, les autres grands marchands internationaux.

\section{ABSTRACT}

Around 1550, thanks to a happy combination of circumstances, P. Assézat found himself in the possession of a great fortune and his social situation became enviable. Nevertheless, his conversion to Calvinism and his active participation to the uprising of the Huguenots in 1562 forced him to exile and exposed him to reprisals (jail). However, it did not prevent him from keeping on supporting the Reformed cause thanks to the international network that his mercantile activity abled him to constitute, notably in the Netherlands. Even after his religious change of position in 1572, he kept on being in close faith to the king of Navarre. And during all this time, he did not neglect his businesses, such as the sale of woad that he delt indifferently with Protestants or Catholics, and thus conforming himself to the common practice of the other great international merchants. 